\title{
Measles outbreak in an asylum-seekers' shelter in Germany: comparison of the implemented with a hypothetical containment strategy
}

\author{
A. TAKLA ${ }^{1,2,3 *}$, A. BARTH ${ }^{4}$, A. SIEDLER ${ }^{1}$, P. STÖCKER ${ }^{1,2,3}$, O. WICHMANN ${ }^{1}$ \\ AND Y. DELERÉ 1 \\ ${ }^{1}$ Immunization Unit, Robert Koch Institute, Berlin, Germany \\ ${ }^{2}$ Postgraduate Training for Applied Epidemiology (PAE), Robert Koch Institute, Berlin, Germany \\ ${ }^{3}$ European Programme for Intervention Epidemiology Training (EPIET), European Centre for Disease \\ Prevention and Control, Stockholm, Sweden \\ ${ }^{4}$ District Health Authority, City of Neumünster, Germany
}

(Accepted 16 November 2011; first published online 12 December 2011)

\begin{abstract}
SUMMARY
In order to contain a measles outbreak in a German asylum-seekers' shelter, serological testing of all residents was performed, followed by selective vaccination of those with negative test results/ not tested. In this paper we describe the outbreak epidemiologically and then compare the implemented strategy with a hypothetical mass vaccination of all individuals unvaccinated or with unknown vaccination status born after 1970 as recommended by the German Standing Committee on Vaccination in terms of potentially avoided cases, logistics, and costs. Three hundred $(70 \%)$ residents participated in the serological testing, of which $39(13 \%)$ were seronegative. In total, 144 individuals were eligible for vaccination, while a mass vaccination would have targeted 359 persons. However, serological testing was time- and personnel consuming and revealed several logistical problems. Its costs amounted to $€ 90000$, double that of mass vaccination that additionally might have avoided three of the eight cases. Mass vaccination seems the preferred measure for measles outbreak control in such settings.
\end{abstract}

Key words: Germany, mass vaccination, measles, outbreak, refugees.

\section{INTRODUCTION}

Measles is one of the most contagious infectious diseases, a viral disease that can cause severe morbidity and complications such as pneumonia or encephalitis. Its case-fatality rate is estimated to be $0 \cdot 2 \%$ in developed countries [1] and 3-5\% in developing countries [2]. A vaccine has been available since 1963 [1], but population vaccination coverage has to exceed $92-95 \%$ to prevent outbreaks [3]. Besides a routine

\footnotetext{
* Author for correspondence: Dr A. Takla, MPH, Immunization Unit, Robert Koch Institute, DGZ-Ring 1, 13086 Berlin, Germany. (Email: TaklaA@rki.de)
}

two-dose measles vaccination strategy in childhood, the German Standing Committee on Vaccination (STIKO) recommends immediate vaccinations in measles outbreak settings. The intervention [i.e. one dose of measles virus (MV)-containing vaccine] should target all persons born after 1970 (with the exception of children aged $\leqslant 6$ months and pregnant women) that are either unvaccinated, received only one vaccination during childhood, or whose immunization status is unknown [4].

In the past 6 years, 19000-43000 persons annually have been seeking asylum status in Germany [5, 6]. They are housed upon their arrival in admittance shelters in all 16 federal states, based on a preset state 
quota for intake per year. Each of these shelters hosts several hundred persons of various ethnicities. Living conditions are crowded and individuals remain for at least 6 weeks up to several months until a final decision regarding their refugee status has been made. The German Asylum Seeker Benefits Law stipulates that asylum seekers are entitled to receive all vaccinations recommended by STIKO for the general German population. The ensuing costs are thereby covered by the responsible asylum-seeker authorities instead of the statutory health insurance funds providing for the general population [7].

During the last years, outbreaks of varicella and measles have been reported in migrant populations or in asylum shelters in Germany $[8,9]$. The STIKO recommendations contain no specific regulations for the management of communicable disease outbreaks in shelters, and on-site management of the outbreak lies with the discretion of the responsible district health authority where the shelter is located. In general, outbreaks in such settings impose unique challenges on local health authorities involving language barriers, cultural differences, potentially dismissive habitus of residents towards public authorities due to previous negative experiences, large family sizes, and unavailability of medical or vaccination records. These challenges become even more severe as containment actions require a rapid response to prevent further cases.

In October-November 2010, a measles outbreak occurred in a shelter in Northern Germany with a total of eight cases in the 427 residents. The shelter usually accommodates about 400 residents from many nations with 4-6 people sharing one room and about 80 people living on each floor. The shelter has a common dining room and hosts a kindergarten and school. A medical post in the shelter grounds performs the statutory medical examinations at intake and provides medical services during weekdays. For identification, the shelter administration issues identity cards upon arrival that include information on name, sex, birth date, shelter entry date and nationality. Children are either added on the mother's or father's identity card.

As available public health resources in Germany are decreasing and measles-mumps-rubella (MMR) vaccine doses are comparatively expensive, local health authorities increasingly consider the alternative of prior serological testing of all shelter residents followed by a selective vaccination for those without protective measles antibodies instead of a mass vaccination. Compatible with this observation, the local health authorities in charge decided upon serological testing and selective vaccinations in this recent measles outbreak.

Previously published studies have focused on the cost comparison of different vaccination strategies and the costs related to individual patients [10-12]. Some studies analysed the comprehensive costs of a measles outbreak [13-15] but did not incorporate the comparison of different outbreak control strategies. The objective of our study was first, to give an epidemiological description of the outbreak and second, evaluate the executed strategy against a hypothetical immediate mass vaccination in terms of potentially avoided cases, logistics, and costs.

\section{METHODS}

\section{Epidemiological description and timeline of the outbreak}

We described the time-course of the outbreak, basic demographics of the shelter population, percentages of residents participating in the voluntary serological testing and vaccination, and seronegativity by age group. The epidemiological description was based on the official residents list and complemented by the review of information on residents' identity cards during the serological testing and vaccination.

\section{Case definition}

A confirmed case was defined as a shelter resident with a laboratory confirmation [positive immunoglobulin (Ig)M, fourfold increase in $\mathrm{IgG}$, or a positive polymerase chain reaction (PCR)] and clinical symptoms ( $\geqslant 3$ days of maculo-papular rash and fever and cough or catarrh or Koplik spots or conjunctivitis) with disease onset between 13 October and 11 December 2010. A suspected case was defined as a shelter resident with the above-mentioned clinical symptoms and disease onset, but without laboratory test results. We assumed every resident to have been exposed to a measles case due to the crowded living conditions at the shelter.

\section{Laboratory analyses and persons eligible for vaccination}

Residents with measles symptoms identified at the shelter's medical post or the local hospital were tested for MV-specific IgM and IgG antibodies, as well as 
MV-specific nucleic acid by PCR at either the local hospital or a large regional laboratory in Hamburg ( $\sim 60 \mathrm{~km}$ from the shelter). In addition, oral swabs of suspected cases were sent for genotyping [16] to the National Reference Center (NRZ) for MMR.

Blood samples of participating shelter residents were sent to and analysed within 1 day at the laboratory in Hamburg. An enzyme-linked immunosorbent assay (ELISA) test (Virotech, Germany) was used to determine $\mathrm{IgG}$ antibody titres. A test was declared positive if titres were $>11$ Virotech units (VE) $[\mathrm{VE}=(\mathrm{OD}$ serum $/ \mathrm{OD}$ cut-off $) \times 10]$, borderline if 9-11 VE, and negative if $<9 \mathrm{VE}$. All residents with a negative or borderline $\mathrm{IgG}$ test result or no test result available and who were not pregnant or aged $\leqslant 6$ months were offered a MMR vaccination.

Immunization cards of all employees working in the shelter were checked to assess their immunization status. Employees without sufficient protection according to STIKO recommendations were offered vaccination.

\section{Evaluation of the two containment strategies}

We evaluated two containment strategies in terms of potentially avoided cases, logistics and costs: serological testing followed by selective vaccination $v s$. a hypothetical mass vaccination immediately starting after the first diagnosed measles case.

\section{Potentially avoided cases by immediate mass vaccination}

We made three assumptions to assess the number of potentially avoided cases by immediate mass vaccination. First, a mass vaccination would have started the day after the first correctly diagnosed measles case (2 November) and therefore 18 days earlier than during the actual outbreak intervention. Second, we assumed the commonly acknowledged mean incubation period for measles from infection to onset of rash of 14 days (range $7-18$ days) $[1,2,17]$ to calculate the assumed time-point of infection. Third, we assumed that a mass vaccination would have targeted all persons included in the STIKO recommendations. Based on those assumptions, we reviewed all cases occurring after 2 November.

\section{Outbreak intervention logistics}

We closely documented the logistics of the conducted outbreak intervention. The goal of this precise description was to evaluate the operability and specific challenges encountered during the serological testing strategy and then compare the findings to anticipated logistical steps and infrastructural needs in a mass vaccination.

Name and date of birth on each identity card were double-checked with the information on the official residents list, name on the test tube, and the laboratory request form prior to blood drawing. Blood samples were collected at the end of each intervention day and analysed immediately, even on the weekend. Names on the test tubes and the laboratory request forms were handwritten and then translated into the laboratory computer system. We matched incoming test results with the residents list of persons that came for testing. Shelter staff sought out residents individually to communicate the test findings. If the test result was seronegative or borderline, residents were asked to attend for vaccination during the following 2 days. Further, all residents who opted not to participate in the serological testing were offered a vaccination. If persons could not be contacted personally, a message was left.

\section{Cost assessment of the two strategies}

We assessed medical and non-medical costs for both strategies by reviewing outbreak-associated bills. Medical costs included MMR vaccines, serological testing, hospitalization, and costs for medical staff. Non-medical costs incorporated personnel costs of the shelter administration, translators, a police-enforced curfew, and costs associated with an instantaneous shelter transfer stop for new incoming or outgoing asylum seekers. The bill review was supplemented by stakeholder interviews (local health authorities, shelter administration, shelter's medical post, laboratory staff, and vaccine manufacturer). Information concerning the hypothetical mass vaccination was extrapolated from actual outbreak figures and stakeholder interviews. For the transfer stop we assumed the moratorium to end about $2 \frac{1}{2}$ weeks after the first case occurred. It was not possible to assign all personnel costs in exact detail to each single logistical step of the serological testing strategy. Therefore, based on the observed length of the serological testing strategy of 41 days (1 November to 11 December), we calculated the personnel costs for the local health authority as a fraction (18 days, 1-18 November). Translator-associated costs were listed according to the smaller number of required assignments. 
Personnel costs for the medical shelter's post were based on experiences during the intervention: total number to be vaccinated and different vaccination times needed per child $(\sim 15 \mathrm{~min})$ and adult ( $\sim 10 \mathrm{~min})$. In addition, we added medical shelter post personnel costs related to other logistical tasks again as a time fraction. Personnel costs of the shelter administration were calculated by the shelter administration head with knowledge of the transfer stop duration.

\section{RESULTS}

\section{Epidemiological description and timeline of the measles outbreak}

A total of eight measles cases occurred over a period of about 4 weeks in the 427 shelter residents, who originated from 18 different nations (Afghanistan $25 \%$, Serbia $17 \%$, Macedonia $10 \%$, Iraq $10 \%$, Iran $7 \%$, Syria $6 \%$, Turkey $5 \%$, and each of the following below 5\%: Azerbaijan, Somalia, Kosovo, Russian Federation, Eritrea, Algeria, Vietnam, Armenia, Yemen, Ghana, India). A total of $254(59 \cdot 2 \%)$ of the residents were male and 172 female $(40 \cdot 3 \%)$. The median age was 23 years (range 14 days to 67 years). There were $145(34 \%)$ children aged $\leqslant 18$ years, 229 (53.9\%) persons aged 19-40 years, and 51 (12\%) aged $>40$ years. No information on nationality, age or sex was available for two long-term absent residents.

The median age of the eight cases was 19 years (range 4-30 years). Four $(50 \%)$ were female, two of them were pregnant. Six $(75 \%)$ out of eight cases had to be hospitalized with an average duration of 4 days. All eight cases were confirmed measles cases: four were confirmed by PCR, three by MVspecific IgM, and one by an increase in MV-specific IgG titre. Genotyping identified MV genotype D4 Hamburg [16] as the causative strain of the outbreak.

The timeline of the outbreak with date of symptom onset in cases is presented in Figure 1. The first measles case was a 30-year-old woman with disease onset on 21 October, whose rash was misdiagnosed by a physician as a generalized allergy. On 1 November, her 5-year-old son (case 2) developed a rash that lead to the correct diagnosis of measles. Case 3 was a 19-year-old pregnant woman (8th month), who was diagnosed with a measles infection on 3 November and went into fever-induced labour on the same day. The child was given immunoglobulins on day 3 postpartum and showed no signs of infection later. Case 4 ( 4 years), case 7 (6 years) and case 8 (25 years) lived on the same floor as the first two cases. Case 8 was 8 months pregnant, and the mother of cases 4 and 7. Cases 5 and 6 were young adults of different nationalities that had no different epidemiological link to the other cases than using the common shelter facilities. It could not be resolved where the index case had contracted her measles infection. She and her family had entered Germany on 4 October from Hungary and arrived in the shelter 10 days later, after having passed through two other asylum shelters in a different federal state in Germany. No associated measles cases were reported from those two shelters or the surrounding health districts.

Simultaneously with the report of the first correctly diagnosed measles case (case 2), the local health authorities issued an immediate transfer stop for the shelter that lasted from 1 November until 11 December. In addition, a police-enforced curfew for all residents (enforcing shelter residents not to leave the compound) was declared on 1 November but proved unmanageable and was thus ended after $27 \mathrm{~h}$. The local health authorities requested outbreak support from the Robert Koch Institute (RKI) on 15 November. The voluntary serological testing and selective vaccinations took place within the following 5 days (Fig. 1).

Forty-five $(31 \%)$ out of 144 shelter employees received a MMR vaccination due to unknown or incomplete vaccination status. None of the employees contracted a measles infection. No measles cases were reported outside the shelter during the outbreak period, but it cannot be ruled out that cases occurred without being reported.

\section{Serological test results and persons eligible for vaccination}

None of the residents was able to provide immunization documentation. Three hundred $(70 \cdot 3 \%)$ out of 427 shelter residents participated in the voluntary serological testing, of whom 39 (13\%) tested negative for MV-specific IgG. Children aged $<9$ months were not tested for MV-specific antibodies. Figure 2 shows the proportion of seronegative persons by age group. The attack rate in all residents was $1.9 \%(8 / 427)$, and in seronegative individuals including cases it was $17 \%$ (8/47).

Regarding non-tested individuals, a total of 166 persons were considered for vaccination. However, 


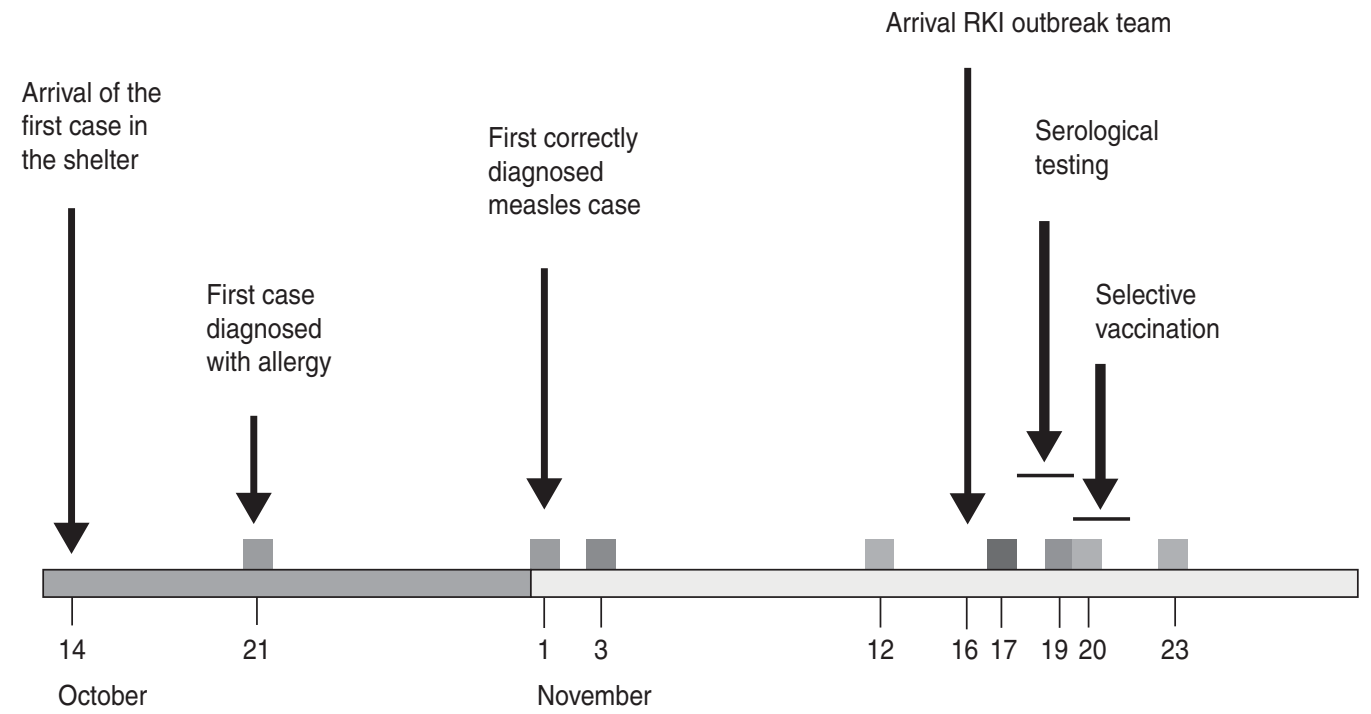

Fig. 1. Timeline of the measles outbreak in an asylum-seekers' shelter in Northern Germany, October/November 2010. Identical shading indicates members of the same family.

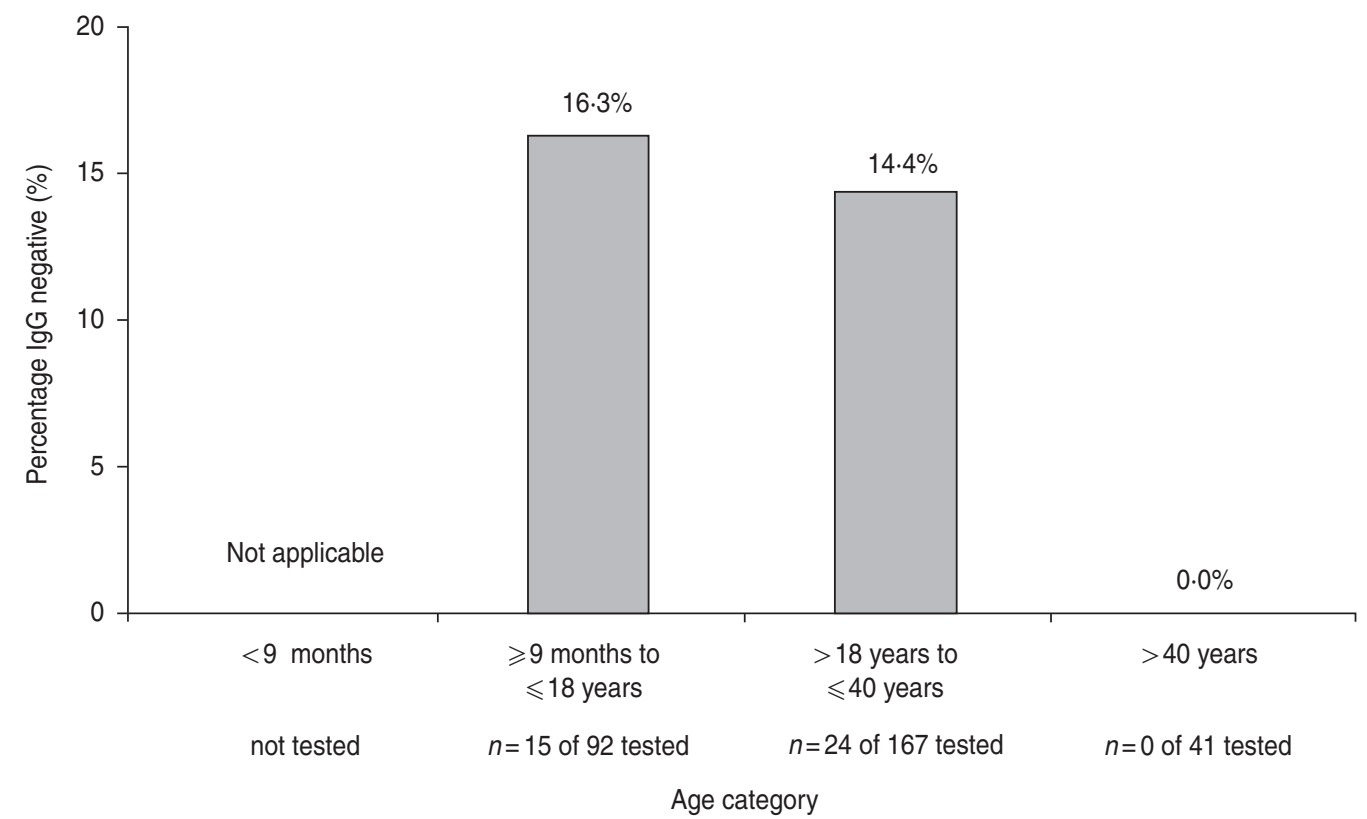

Fig. 2. Percentage of measles IgG seronegatives among those tested $(n=300)$ by age group during a measles outbreak in an asylum-seekers' shelter in Northern Germany, 2010.

$22(13 \cdot 3 \%)$ were or could not be offered a vaccination due to pregnancy $(n=7)$, age $\leqslant 6$ months $(n=4)$, having become a confirmed case $(n=4)$, being an unconfirmed but suspected case $(n=3)$, long-term absenteeism from the shelter $(n=3)$, or refusal of vaccination $(n=1)$. Of the remaining 144 eligible individuals, $101(70 \cdot 1 \%)$ were vaccinated during a 2-day vaccination operation, including two children aged between $>6$ and $\leqslant 8$ months. In the following week, another seven $(4.9 \%)$ residents requested vaccination at the shelter's medical post.

\section{Potentially avoided cases by immediate mass vaccination}

Based on the current STIKO guidelines, residents' age distribution, and individual reasons for exclusion, $359 / 427(84 \%)$ residents would have been eligible for 


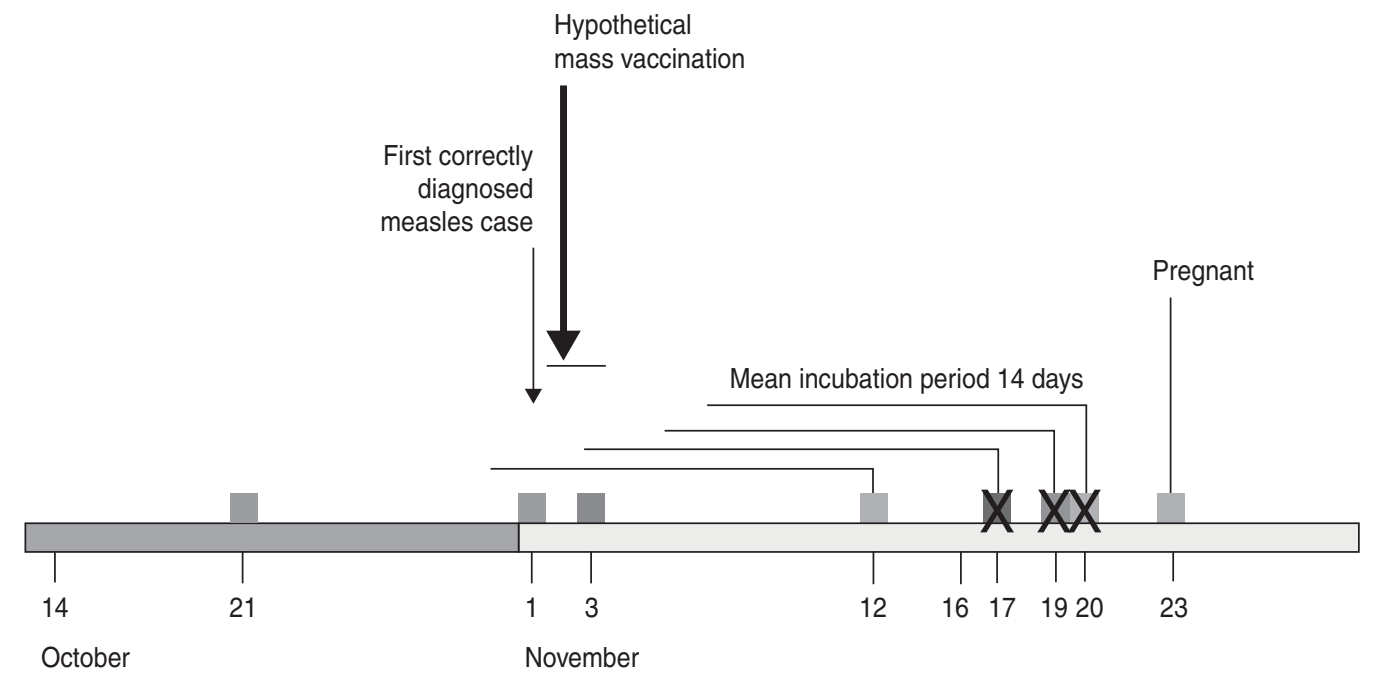

Fig. 3. Potentially avoided measles cases by a hypothetical immediate mass vaccination during an outbreak in an asylumseekers' shelter in Northern Germany, 2010. Cases are represented by squares, potentially avoided cases are marked with a black cross $(\mathrm{X})$.

vaccination. Under our previously described assumptions with a mean incubation period of 14 days, an immediate mass vaccination might have avoided three cases (Fig. 3). Considering a range of 7-18 days, the figure did not change substantially with 3-4 possibly avoided cases.

\section{Outbreak intervention logistics}

It often proved difficult to assign the identity card name to the corresponding name on the official list due to transpositions of first and last name, serious spelling mistakes, and middle names being added as another last name or vice versa. Furthermore, birthdates sometimes did not match between the two sources. A number of people were not listed at all, yet they possessed a shelter-issued identity card. Another group not listed were children that were additionally not registered on a parent's identity card.

In five $(2 \%)$ cases we detected names on test tubes that were different from the person from whom blood was to be drawn. During the matching of the laboratory results with our residents list of serological testers, we found in $6 \%(17 / 300)$ new serious spelling mistakes or transpositions of first and last names that had occurred during laboratory transcription.

Necessary resident contacts added up to four encounters to communicate findings and carry out intervention procedures for the serological testing strategy (information for residents, blood collection, information about the results, and possible vaccination), compared to two for a mass vaccination (information on the vaccination procedure and risks, and the vaccination itself).

\section{Cost assessment of the two strategies}

Medical and non-medical costs for the two strategies are listed in Table 1. The serological testing with selective vaccination amounted to $\sim € 90000$ and was nearly double the costs of a hypothetical mass vaccination $(\sim € 47000)$. The shelter transfer stop substantially contributed with $\sim € 34000$ to the total costs of the serological testing strategy, compared to $\sim € 4000$ in the hypothetical mass vaccination. Reviewed bills and the interview with the head of the shelter administration revealed that those costs increase exponentially over time instead of linearly.

\section{DISCUSSION}

The main aim of our study was to compare two containment strategies in terms of potentially avoided cases, logistics, and costs. Our findings related to these three aspects clearly favour a mass vaccination strategy in such a setting.

A very important evaluation factor is the number of potentially avoided cases, as they are the main goal of each outbreak containment effort. Applying the mean incubation period of 14 days until rash onset, the immediate mass vaccination might have avoided three of the eight cases. Considering the time until immunity 
Table 1. Comparison of outbreak-associated costs for the two containment strategies during a measles outbreak in an asylum-seekers' shelter in Northern Germany, 2010

\begin{tabular}{|c|c|c|c|c|}
\hline & \multicolumn{2}{|c|}{$\begin{array}{l}\text { Serological testing and selective } \\
\text { vaccination }\end{array}$} & \multicolumn{2}{|l|}{ Mass vaccination } \\
\hline & Number of units & Total $(€)$ & Number of units & Total $(€)$ \\
\hline \multicolumn{5}{|l|}{ Medical costs } \\
\hline \multicolumn{5}{|l|}{ Resources } \\
\hline Serological testing* & $N=300$ & $3330 \cdot 00$ & & \\
\hline \multicolumn{5}{|l|}{ Vaccines $\dagger$} \\
\hline Shelter residents & $N=144$ & $5119 \cdot 20$ & $N=359$ & $12762 \cdot 45$ \\
\hline Shelter staff & $N=45$ & $1599 \cdot 75$ & $N=45$ & $1599 \cdot 75$ \\
\hline \multicolumn{5}{|l|}{ Hospitalizations } \\
\hline Case 2 & $3 \mathrm{~d}$ & $1653 \cdot 42$ & $3 \mathrm{~d}$ & $1653 \cdot 42$ \\
\hline Case 3 & $6 \mathrm{~d}$ & $1760 \cdot 66$ & $6 \mathrm{~d}$ & $1760 \cdot 66$ \\
\hline Case 4 & $5 \mathrm{~d}$ & $1743 \cdot 42$ & $5 \mathrm{~d}$ & $1743 \cdot 42$ \\
\hline Case 5 & $3 \mathrm{~d}$ & $1427 \cdot 15$ & & \\
\hline Case 7 & $4 d$ & $1563 \cdot 42$ & & \\
\hline Case 8 & $3 \mathrm{~d}$ & $1518 \cdot 42$ & $3 \mathrm{~d}$ & $1518 \cdot 42$ \\
\hline \multicolumn{5}{|l|}{ Personnel costs } \\
\hline Local health authority & $211 \mathrm{ht}$ & $10516 \cdot 70$ & $91 \mathrm{ht}$ & $4525 \cdot 86$ \\
\hline Shelter medical post & $519 \mathrm{ht}$ & $15439 \cdot 75$ & $277 \mathrm{ht}$ & $7854 \cdot 69$ \\
\hline \multicolumn{5}{|l|}{ Non-medical costs } \\
\hline \multicolumn{5}{|l|}{ Resources } \\
\hline Shelter transfer stop & $41 \mathrm{~d}$ & $33807 \cdot 23$ & $18 \mathrm{~d}$ & $3926 \cdot 30$ \\
\hline \multicolumn{5}{|l|}{ Personnel costs } \\
\hline Shelter administration & $77 \mathrm{~h} \ddagger$ & $3865 \cdot 60$ & $70 \mathrm{~h} \ddagger$ & $3607 \cdot 45$ \\
\hline Translators & & $2563 \cdot 66$ & & $2299 \cdot 48$ \\
\hline Police-enforced curfew (27 h) & $108 \mathrm{~h}$ & $4428 \cdot 00$ & $108 \mathrm{~h}$ & $4428 \cdot 00$ \\
\hline Total & & $90336 \cdot 38$ & & $47679 \cdot 90$ \\
\hline
\end{tabular}

d, Days; h, hours.

* Unitary cost: $€ 11 \cdot 10$.

$\dagger$ Unitary cost: $€ 35 \cdot 55$.

\$ Different wages per hour according to position, only total number of hours given.

in vaccinated individuals develops, two recent studies have shown an effective measles post-exposure prophylaxis (PEP) of $90.5 \%$ and $100 \%$, respectively, if conducted within $72 \mathrm{~h}[18,19]$. In individuals who developed measles despite PEP, two other studies observed a maximum time-interval from receiving PEP to the development of measles of $\sim 10$ days $[20,21]$. For a conservative estimate we assumed that case 4 would still have developed the disease. However, based on the data from the studies cited above, it is quite likely that case 4 would also have been avoided by mass vaccination or at least benefited from vaccination, as even vaccination during the incubation period might mitigate measles symptoms [22]. Case 8 was pregnant and would not have been vaccinated at any point. However, a comprehensive vaccination of the shelter community (including her two children, later cases 4 and 7) might have granted her herd protection, possibly preventing her from becoming infected.

Logistics associated with the serological testing proved to be challenging in this special setting. A good laboratory infrastructure as in our study is not always available, and less timely receipt of results prolongs the time needed to complete subsequent vaccinations. Furthermore, the proportion of children in the shelter was high. In this age group, blood drawing and vaccinations on average require more time, and a second, needle-associated intervention (if seronegative) is usually more stressful for children than for adults. Only two-thirds of the residents participated in the voluntary serological testing. In addition to the seronegatives, the remaining 127 persons were therefore eligible for vaccination due to their unknown serological status. Finally, the selective vaccination strategy required four contacts between 
residents and the intervention team compared to two in a mass vaccination. The more contacts that are required, the higher the chance that residents do not complete all steps necessary for a successful containment intervention.

Overall costs associated with the outbreak were high, even though the outbreak consisted of only eight measles cases. Costs for the serological testing and selective vaccination strategy were nearly double those anticipated for the hypothetical mass vaccination. About one third of the costs in the serological testing strategy were attributable to the 6 weeks shelter transfer stop. As a short closure of 2-3 weeks can be compensated for with personnel resources at hand, longer closures require extra resources after reopening: besides the timely resumption of a high volume of delayed hearings, a high number of asylum seekers has to be admitted in a short time period to be able to fulfil the preset yearly intake quota. Furthermore, personnel costs made for substantial relative cost differences in both containment strategies.

In our study we compared two interventions that would have taken place at different time points. However, local health authorities are very likely to decide whether to conduct serological testing with selective vaccination or an immediate mass vaccination referring to the same point in time. If so, there are several conceptual thoughts to consider. In principle, the duration of both interventions depends on resource- and infrastructure-related factors. The duration of blood sampling and vaccination is associated with the number of medical staff available, the number of residents in the shelter, and the proportion of children among residents. For the serological testing strategy, the expenditure of time is additionally related to the expected number of seronegatives with subsequent vaccination among shelter residents. The length of laboratory analysis is determined by the testing capacity of the laboratory and by the duration and frequency of specimen shipments to the laboratory (each day $v s$. once only). Thus, the time between diagnosis of the index case and vaccination of the last eligible person can be several days longer for the serological testing strategy than in our outbreak, increasing the risk of secondary measles cases. If personnel resources and infrastructure are limited, concentrating on an immediate mass vaccination will increase the chances of staying within the desired $72 \mathrm{~h}$ for PEP. Of course, timelines of serological testing can be enhanced by increasing the deployed personnel in the intervention. However, increasing the involved personnel would lead to a substantial increase in personnel-related costs-contradicting one of the common arguments that serological testing is more cost-effective compared to an immediate mass vaccination.

During a mass vaccination campaign, some previously vaccinated individuals without immunization documentation will consequently receive an unnecessary vaccination. Multiple vaccinations often raise concerns about tolerability and adverse effects. However, there are no references in the literature that support those concerns [4, 23, 24]. One study found no increased morbidity if $\mathrm{MV}$-incubated persons receive a MMR vaccination [22].

The relatively few cases and the prolonged outbreak influenced the local health authorities' decision for the containment strategy of serological testing and selective vaccination. Possible reasons for the small number of cases despite crowded living conditions and a measles basic reproductive number of 12-16 [3] might be that with $85 \%$ protected individuals the effective reproductive number was considerably lower and that more heterogeneous rather than homogeneous mixing occurred. Both conditions have the potential to lead to prolonged and less explosive outbreaks.

Seroprevalence studies for vaccine-preventable diseases in non-outbreak situations in refugees or asylum seekers have been previously conducted [25, 26]. However, characteristics and demographics of persons fleeing a specific country can change quickly over time depending on the situation in the country of origin. At the shelter level, the number of immune individuals depends greatly on the composition of nationalities in the shelter, the demographics of the citizens as well as individual immunization programmes and circulation of the wild virus in each of the countries of origin. Therefore, seroprevalence studies in these settings remain a snapshot in time with limited value for translatability and thus should not be used as a basis for selective vaccination interventions in outbreaks. Nonetheless, our study as well as the other surveys demonstrates that the proportion of individuals with measles immunity is likely below $95 \%$ in asylum shelters or refugee settings. Hence, outbreaks can easily occur if the virus is introduced.

There are several limitations to our study. MMR vaccine prices are specific to Germany and can be more expensive than in other countries (e.g. €35.50 in Germany vs. $\sim € 13$ for children and $\sim € 23$ for adults in the USA) [27, 28]. Similarly, personnel costs and costs related to the shelter transfer stop are 
country-dependent, composed differently or might not apply. Therefore, our study might have limited transferability across countries. In addition, our study is specific for the setting of an asylum shelter or similar refugee camps where immunization records are generally not available and logistical problems due to language barriers or cultural differences might have a higher impact. Nonetheless, our study provides important insights and challenges for the control of an institutional outbreak of measles which involves refugees and asylum seekers. It also draws attention to a probably previously under-appreciated setting where Germany - among other issues - is facing difficulties in its efforts to achieve measles elimination by 2015 .

One solution to generally prevent future outbreaks in asylum shelters is to improve vaccination practices routinely offered at first intake. Whereby the statutory medical examination taking place within the first days of entry would be a reasonable and easy to execute option for routine vaccinations. In our case, a vaccination of the index person and her family at intake in the very first asylum shelter after arrival in Germany on 4 October would have most likely prevented the later outbreak. However, the wide implementation of this policy is highly dependent on available staff and resources in the local health offices and shelter medical posts. In the affected shelter, only $84 \%$ of the children were protected against measles, compared to the one-dose measles vaccination coverage at school entry in Germany in 2009 where the figure was 96.1\% [29]. As Germany is responsible for the health of persons that have claimed asylum status on its territory and has committed to the WHO goal of measles elimination in Europe by 2015, improved implementation of routine vaccination at first intake could be helpful towards preventing future outbreaks in asylum shelters and the subsequent spread of measles into communities outside these settings.

\section{ACKNOWLEDGEMENTS}

The authors thank Dr Karin Paeth and Susanne Jessen (District Health Authority, Neumünster), and Dr Anette Junghans and her staff at the shelter's medical post for their indispensable support during the outbreak interventions. We also thank Ulf Döhring, Elisabeth Zimmermann, Ralf Krause, and Monika Krause of the shelter administration (Landesamt für Ausländerangelegenheiten, Neumünster) for their assistance during the outbreak management and the provision of cost data and other background information; PD Dr Annette Mankertz and Dr Sabine Santibanez (National Reference Center Measles Mumps Rubella, RKI, Berlin) for the genotyping of specimens; Dr Mohrmann and his staff (Labor Lademannbogen, Hamburg) for their rapid provision of serological test results; and all residents of the shelter for supporting this investigation with their participation. Finally, we acknowledge Sandra Dudareva (RKI) for her practical assistance during the outbreak and Dr Katharina Alpers (PAE coordinator, RKI) for her intellectual input in writing this report.

\section{DECLARATION OF INTEREST}

None.

\section{REFERENCES}

1. Pink Book Chapter 12. Measles (http://www.cdc.gov/ vaccines/pubs/pinkbook/downloads/meas.pdf). Accessed 14 July 2011.

2. Heymann D (ed.). Control of Communicable Diseases Manual, 19th edn. Washington, DC: American Public Health Association, 2008, pp. 402-408.

3. Anderson RM, May RM. Immunisation and herd immunity. Lancet 1990; 335: 641-645.

4. Robert Koch Institute. Recommendations by the German Standing Committee on Vaccination (STIKO)/ July 2010 [in German]. Epidemiologisches Bulletin 2010; 30: 279-298.

5. Applications for asylum 2010. (http://www.bamf.de/cln 111/SharedDocs/Pressemitteilungen/DE/2011/1101170001-press emitteilung-bmi.html). Accessed 10 February 2011.

6. Report on migration 2009. (http://www.bmi.bund.de/Shared Docs/Downloads/DE/Broschueren/2011/Migrationsbericht_ 2009_de.pdf?_blob=publicationFile). Accessed 3 May 2011.

7. Asylum Seeker Benefits Law. $§ 4$ Benefits during disease, pregnancy and childbirth (http://bundesrecht.juris.de/ asylblg/_4.html). Accessed 26 July 2011.

8. Gold H. About a measles outbreak among migrants in Munich [in German]. Epidemiologisches Bulletin 2010; 50: $501-504$.

9. Niedermeier A, Dreweck C. Varicella: on a cluster among Somali asylum seekers in two shelters in Munich [in German]. Epidemiologisches Bulletin 2010; 48: 480-482.

10. Davis RM, et al. A cost-effectiveness analysis of measles outbreak control strategies. American Journal of Epidemiology 1987; 126: 450-459.

11. Shiell A, et al. Cost-effectiveness of measles outbreak intervention strategies. Australian and New Zealand Journal of Public Health 1998; 22: 126-132.

12. White CC, Koplan JP, Orenstein WA. Benefits and costs of immunization for measles, mumps and 
rubella. American Journal of Public Health 1985; 75 : 739-744.

13. Wichmann O, et al. Futher efforts needed to achieve measles elimination in Germany: results of an outbreak investigation. Bulletin of the World Health Organization 2009; 87: 108-115.

14. Dayan GH, et al. The cost of containing one case of measles: the economic impact on the public health infrastructure - Iowa, 2004. Pediatrics 2005;116:e1-e4.

15. Sugarman DE, et al. Measles outbreak in a highly vaccinated population, San Diego, 2008: role of the intentionally undervaccinated. Pediatrics 2010; 125: 747-755.

16. Kremer JR, et al. High genetic diversity of measles virus, World Health Organization European Union Region, 2005-2006. Emerging Infectious Diseases 2008; 14: $107-114$

17. World Health Organization. Measles vaccines: WHO position paper. Weekly Epidemiological Record 2009; 84: $349-360$

18. Sheppeard V, et al. The effectiveness of prophylaxis for measles contacts in NSW. NSW Public Health Bulletin 2009; 20: 81-85.

19. Barrabeig I, et al. Effectiveness of measles vaccination for control of exposed children. Pediatric Infectious Disease Journal 2011; 30: 78-80.

20. Rice $\mathbf{P}$, et al. MMR immunisation after contact with measles virus. Lancet 2004; 363: 569-570.

21. McKeever $\mathbf{V}$, et al. Postexposure prophylaxis, isolation, and quarantine to control an import-associated measles outbreak - Iowa, 2004. Morbidity and Mortality Weekly Report 2004; 53: 969-971.

22. Sakuta H, Sawada S, Kuroki Y. Severity of measles among patients with incidental postexposure vaccination. Japanese Journal of Infectious Diseases 2008; 61: 304-306

23. Watson JC, et al. Measles, mumps, and rubellavaccine use and strategies for elimination of measles, rubella, and congenital rubella syndrome and control of mumps: recommendations of the Advisory Committee on Immunization Practices (ACIP). Morbidity and Mortality Weekly Report. Recommendations and Reports 1998; 47: 1-57.

24. Plotkin SA, Orenstein WA, Offit PA (eds). Vaccines, 5th edn. Philadelphia: Saunders, 2008, pp. 353-398.

25. Barnett ED, Christiansen D, Figueira M. Seroprevalence of measles, rubella, and varicella in refugees. Clinical Infectious Diseases 2002; 35: 403-408.

26. Greenaway C, et al. Susceptibility to measles, mumps, and rubella in newly arrived adult immigrants and refugees. Annals of Internal Medicine 2007; 146: 20-24.

27. Rote Liste 2010 (http://www.rote-liste.de). Accessed 12 February 2011.

28. CDC Vaccine Price List (http://www.cdc.gov/vaccines/ programs/vfc/cdc-vac-price-list.htm). Accessed 20 June 2011.

29. Robert Koch Institute. Vaccination coverage at school entry in Germany 2009 [in German]. Epidemiologisches Bulletin 2011; 16: 125-129. 